\begin{tabular}{|c|c|c|c|c|c|}
\hline Revista Clío América & ISSN: 1909-941X & Vol. 12 & No. 23 & enero - junio de 2018 & 98 - 108 \\
\hline
\end{tabular}

\title{
Gestión logística en el sector textil-confección en colombia: retos y oportunidades de mejora para la competitividad
}

\author{
Logistics management in the textile-clothing sector in Colombia: challenges and \\ opportunities for the improvement of competitiveness
}

RESUMEN: El presente artículo tiene como objetivo revisar y analizar la problemática actual del sector textil - confección, diseño y moda en Colombia, derivada de factores externos relacionados con la situación socioeconómica del país, las políticas de importación y exportación, la competencia desleal, las constantes reformas tributarias, entre muchos otros que pueden hacer vulnerable uno o más eslabones en la cadena de suministro. Con el fin de identificar el rol de la logística en este sector empresarial y plantear alternativas de productividad y competitividad, se realiza un análisis minucioso del marco teórico disponible, se procede con la realización de entrevistas a empresarios y se establece un estado del arte de la gestión logística. Como resultado, se plantea un contexto de la situación actual del sector y se proponen acciones y posibles aportes desde la logística que aumenten la productividad y competitividad de las empresas que pertenecen a este sector económico.

Palabras clave: logística - SCM - competitividad - industria textil-confección.

JEL: L67, M11, M21

ABSTRACT:This article aims to review and analyze the current problems of the textile sector - clothing, design and fashion in Colombia, derived from external factors related to the socioeconomic situation of the country, import and export policies, unfair competition, continued tax reforms, among many others that can make vulnerable one or more links in the supply chain. In order to identify the role of logistics in this business sector, and to propose alternatives for productivity and competitiveness, a thorough analysis of the available theoretical framework is carried out, interviews with businessmen are conducted and a state of art of logistics management is established. As a result, a context of the current situation of the sector, as well as actions and possible contributions are proposed from the logistics point of view to increase the productivity and competitiveness of the companies that belong to this economic sector.

Keywords: Logistics - SCM - Competitiveness - Textile-Clothing Industry.

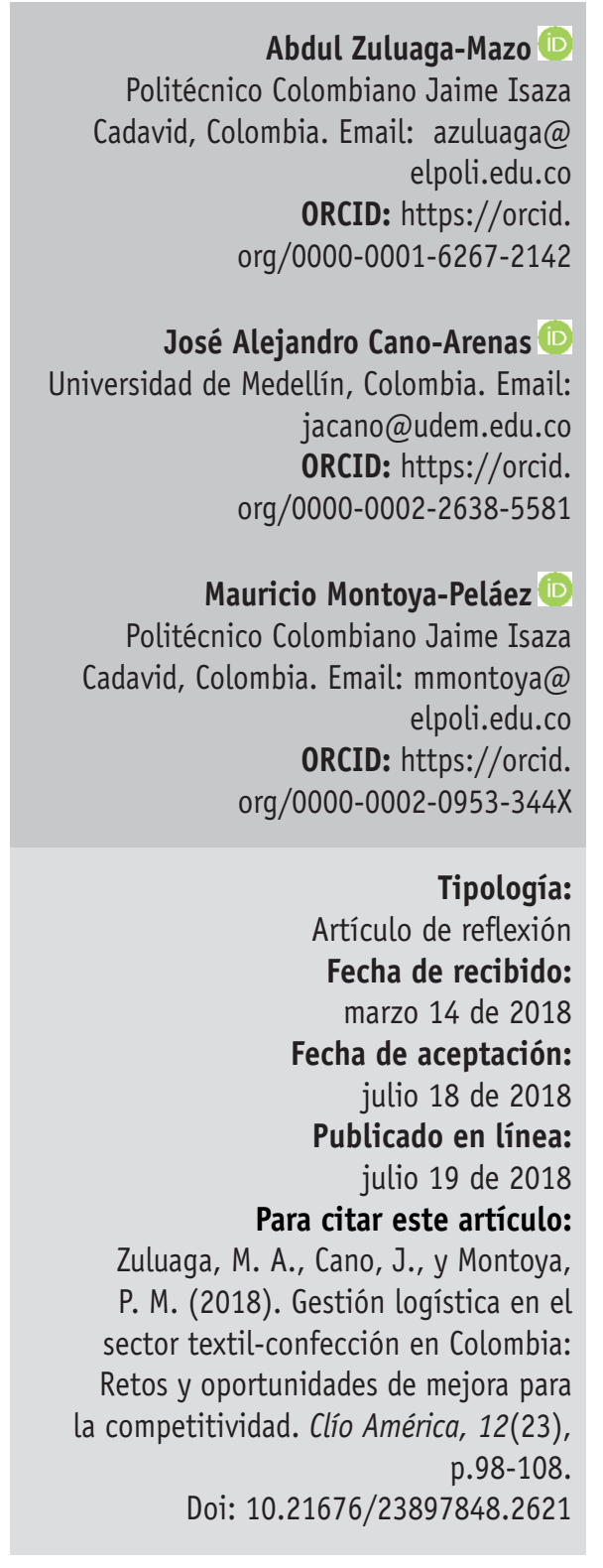




\section{INTRODUCCIÓN}

El sector textil-confección, diseño y moda en Colombia ha presentado múltiples dificultades en los últimos años. Su productividad y competitividad se han visto golpeadas por la coyuntura económica que atraviesa el país. Varias reformas tributarias, contracción de la demanda, competencia desleal, contrabando, lavado de activos; son solo algunas de las variables de peso que afectan al sector. Adicional a esto, las cadenas de suministro globales de textiles y confección deben enfrentar hoy en día los altos costos de energía y ambientales, y enfrentar los requerimientos del mercado que demanda una gran variedad de productos con ciclos de vida cada vez menores (Cooper, 2010). Estos cambios en la industria de la confección han motivado el rediseño de las estructuras organizacionales y productivas, con el fin de alcanzar niveles satisfactorios de competitividad para permanecer en el mercado. Por lo tanto, desde un enfoque logístico, de integración de procesos y de competitividad de las cadenas del sector, se hace urgente plantear en el sector textil-confección estrategias de planificación de la demanda, esquemas colaborativos, alianzas estratégicas, manejo estratégico de inventarios, estrategias de liquidez, gestión adecuada de compras, entre otros, que redunden en la generación de valor y enfrenten la intensa competencia tanto en el mercado nacional e internacional (Ramdass y Pretorius, 2011).
Debido a esto, en el presente artículo se realiza un análisis a la problemática del sector textilconfección, diseño y moda desde el punto de vista logístico, y de manera propositiva se plantean acciones que buscan mejorar los índices de rentabilidad y valor para las empresas que pertenecen a esta industria. Para esto, en la siguiente sección se presenta una contextualización teórica sobre la logística en la empresa, la gestión de la cadena de suministro y tendencias en logística tal como la logística 4.0. Seguido de esto se presenta el esquema metodológico propuesto en el artículo, para así realizar en la tercera sección una contextualización del sector textil y confección en Colombia, y presentar en la cuarta sección los hallazgos y discusiones. Finalmente, en la quinta sección se presentan las conclusiones y propuestas para mejorar la competitividad del sector textil-confección en Colombia.

\section{Logística en la empresa}

Las actividades logísticas pueden presentar variaciones o modificaciones de una empresa a otra; sin embargo, las actividades que se reseñan a continuación, simbolizan lo que habitualmente se puede hallar en cualquier tipo de organización (Ballou, 2004). Las actividades que podrían ser señaladas como las fundamentales en un enfoque logístico, son las siguientes: servicio al cliente, el transporte, la administración de inventarios y el procesamiento de órdenes. En la Tabla 1 se resumen estas actividades con las funciones principales que se llevan a cabo.

\section{Tabla 1.}

\section{Actividades y funciones fundamentales de la logística}

\section{SERVICIO AL CLIENTE}

\section{(En cooperación con marketing)}

1. Determinar las necesidades del cliente y requerimientos de servicios logísticos

2. Determinar respuesta de servicio al cliente

3. Fijar niveles de servicio

\section{TRANSPORTE}

1. Interfaces entre órdenes de venta e inventarios

2. Métodos de transmisión de información

3. Reglas para efectuar pedidos 


\section{PROCESAMIENTO DE ÓRDENES}

1. Políticas de inventarios

2. Pronósticos de ventas a corto plazo

3. Mezcla de productos y puntos de almacenamiento

4. Número tamaño y localización de los puntos de almacenamiento

5. Estrategias (push, pull, jit, etc.)

\section{ADMINISTRACIÓN DE INVENTARIOS}

1. Selección de modos de transporte

2. Fletes

3. Rutas de transporte

4. Listado de programación de vehículos

5. Selección de equipos

6. Procesamiento de pedidos

7. Evaluación y auditorías

Fuente: (Ballou, 2004)

Estas actividades fundamentales se apoyan en otras tareas no menos importantes que, en ciertas eventualidades, pueden llegar a ser tan críticas como las principales. Estas son las conocidas actividades de soporte, entre las que caben re- saltar: almacenamiento, manejo de materiales, compras, empaque y embalaje, producción y mantenimiento de la información; las cuales se describen en la Tabla 2, con base en los aportes de Ballou (2004).

\section{Tabla 2.}

\section{Actividades de Soporte de la Logística}

\begin{tabular}{|l|l|}
\multicolumn{1}{|c|}{ ACTIVIDAD } & \multicolumn{1}{c|}{ FUNCIONES } \\
\hline Almacenamiento & 1. Determinación de espacios \\
\hline 2. Diseño de equipos y muelles \\
\hline 3. Configuración del almacén \\
\hline 4. Colocación de inventarios \\
\hline 1. Selección de equipos \\
\hline 2. Políticas de reemplazos de equipos \\
\hline 3. Procedimientos de picking \\
\hline 4. Stock de almacenaje \\
\hline 5. Selección de proveedores \\
\hline 6. Tiempos de entrega \\
\hline 7. Lotes de entrega \\
\hline
\end{tabular}




\section{ACTIVIDAD}

\section{Compras}

Empaque y Embalaje

Mantenimiento de la información

\section{FUNCIONES}

1. Descripción de suministros a adquirir

2. Selección evaluación de proveedores

3. Negociación con proveedores

4. Control de calidad e inspección

5. Entrega de productos al almacén o producción

6. Seguimiento y control de proveedores

1. Diseño para el manejo de productos

2. Diseño para almacenamiento

3. Diseño para protección contra daños y averías

4. Especificaciones de cantidades agregadas

5. Secuencias y tiempos de producción

1. Recopilar, almacenar y manipular

2. Analizar datos

3. Procedimientos de control

Fuente: Ballou (2004).

La distinción que se hace entre actividades fundamentales $y$ actividades de soporte, obedece puntualmente a que las primeras forman parte, en su mayoría, de todos los sistemas logísticos, mientras que la aparición de las otras dependerá del tipo de organización objeto de análisis.

\section{SCM y concepto de cadena de suministro}

La gestión de la cadena de suministro (Supply Chain Management - SCM) se define como la planeación e integración de los flujos de materiales, finanzas, productos e información interna y entre empresas, desde unos proveedores primarios hasta el consumidor final, con el fin de agregar valor a los clientes a través de productos terminados y servicios (Cano, Panizo, García y Rodríguez, 2015). Su aparición dio paso a la creación de un nuevo rango de proceso de negocios que comprende siete etapas: la administración de las relaciones con el cliente, la administración de la demanda, la gestión de pedidos, la administración de flujos de manufactura, el proceso de abastecimiento, el desarrollo de productos y su comercialización (Mentzer, 2004).
Con el desarrollo de las TIC en la última década se han integrado nuevas tecnologías al proceso logístico, lo que ha generado el concepto de Logística 4.0 o Logística Inteligente (Bahadır y Büyüközkan, 2016). Con este nuevo enfoque se pretende dar uso intensivo a la tecnología y a la información con el objetivo de integrar la cadena, es decir, no sólo los proceso internos de la compañía, sino, proveedores, clientes e intermediarios (Domingo, 2016). En consecuencia, una cadena de suministro eficiente puede ser vinculada a la idea de Logística 4,0 con el fin de lograr involucrar toda una serie de modificaciones para el desarrollo de procesos automáticos de sistemas que impliquen un nuevo estilo de administrar los inventarios, haciendo más fácil su manejo desde la llegada a la cadena de abastecimiento como materia prima, hasta la adquisición por parte de un cliente. Todo lo anterior está apoyado en la ayuda de herramientas como la robótica, el Big Data y una visión artificial que permitan una individualización de la producción para que la gestión en movilidad sea lo más eficiente posible (Hülsmann, 2015). Además, se debe mantener una relación directa con los pronósticos de 
la demanda, reducción de inventarios, herramientas para el control de almacenamiento, optimización de rutas, sistemas de localización más novedosos, entre otros.

\section{Logística 4.0}

Justo cuando la industria evoluciona, se identifica la necesidad de desarrollar en las organizaciones una cadena de suministros que se ajuste al entorno cambiante y dinámico de los nuevos tiempos, con prácticas y herramientas innovadoras que permitan evolucionar la logística (Fugate, 2010). Es así como aparece la Logística 4.0 que busca brindar soluciones que integren esas herramientas, prácticas y nuevas tecnologías. Esto implica el uso de herramientas como la identificación por radio frecuencia, el Internet de las cosas (Internet of Things), transacciones de grandes datos (Big Data Analytics), fabricación aditiva, tecnologías usables (Wearable Technologies), vehículos de guiado autónomo y robots humanoides.

\section{METODOLOGÍA}

Para realizar un análisis a la problemática del sector textil-confección, diseño y moda, y plantear acciones para mejorar los índices de rentabilidad y valor para las empresas que pertenecen a esta industria, se propone una metodología compuesta por tres etapas, la cual se presenta en la Figura 1.

\section{Figura 1.}

Diseño metodológico esquematizado

\section{Etapa 1. Revisión del estado del arte en gestión del aprovisionamiento y cadenas de abastecimiento}
Revisión de los conceptos, actividades y procesos de la logística Integral

Definiciones y conceptos del Supply Chain Management (SCM)

Tendencias y nuevas tecnologías asociadas a la logística - Logística 4.0

\section{Etapa 2. Revisión del estado actual del sector textil confección en Colombia}

Caracterización del sector textil confección y sus procesos logísticos

Consulta a fuentes primarias (aplicación de instrumentos para la recolección de información de fuentes primarias)

\section{Etapa 3. Análisis de los resultados}
Hallazgos
Discusión
Conclusiones

\section{Propuestas}

Fuente: elaboración propia. 
En la primera etapa del diseño metodológico se propone una revisión del estado del arte en gestión del aprovisionamiento y cadenas de suministro, donde se hace una exploración de las generalidades de la logística, su evolución hacia la SCM y las tendencias actuales relacionadas con la Logística 4.0. En la segunda etapa se establece un diagnóstico general de la situación actual del sector textil confección en Colombia a través de fuentes primarias y secundarias. En este sentido, se consultaron informes sectoriales de centros de excelencia, institutos del sector textil-confección, diseño y moda, informes del Departamento Nacional de Planeación de Colombia, cámaras de comercio, asociaciones de industriales del país, entre otros. De igual modo, como trabajo de campo se realizaron 14 entrevistas semi-estructuradas, basadas en preguntas abiertas a dirigentes del sector y personal de nivel estratégico directamente relacionado con el tema logístico. En la tercera etapa se genera un análisis de resultados, donde se resume la información recopilada tanto de fuentes primarias como secundarias, con el fin de realizar la discusión de las problemáticas del sector. Posterior a esto, se plantean alternativas que tengan un impacto directo en las dificultades encontradas.

\section{Contextualización Del Sector Textil Y Confección En Colombia}

La industria textil y confección en Colombia representa un sector productivo que cuenta con una trayectoria de más de cien años de experiencia y una cadena de producción consolidada, lo que le ha permitido posicionarse tanto a nivel nacional como internacional, debido principalmente al valor agregado de las telas, a la calidad de las confecciones, a la innovación, a los diseños y a la ocupación de mano de obra local.

Como se muestra en la Figura 2, la industria textil-confección presenta un sistema productivo conformado por productores de fibra de algodón y productores de fibra química, hilanderías, la industria textilalgodón, la industria textil-lanera y la industria de la confección.

\section{Figura 2.}

Sistema Productivo de la Industria Textil - Confección Colombiana

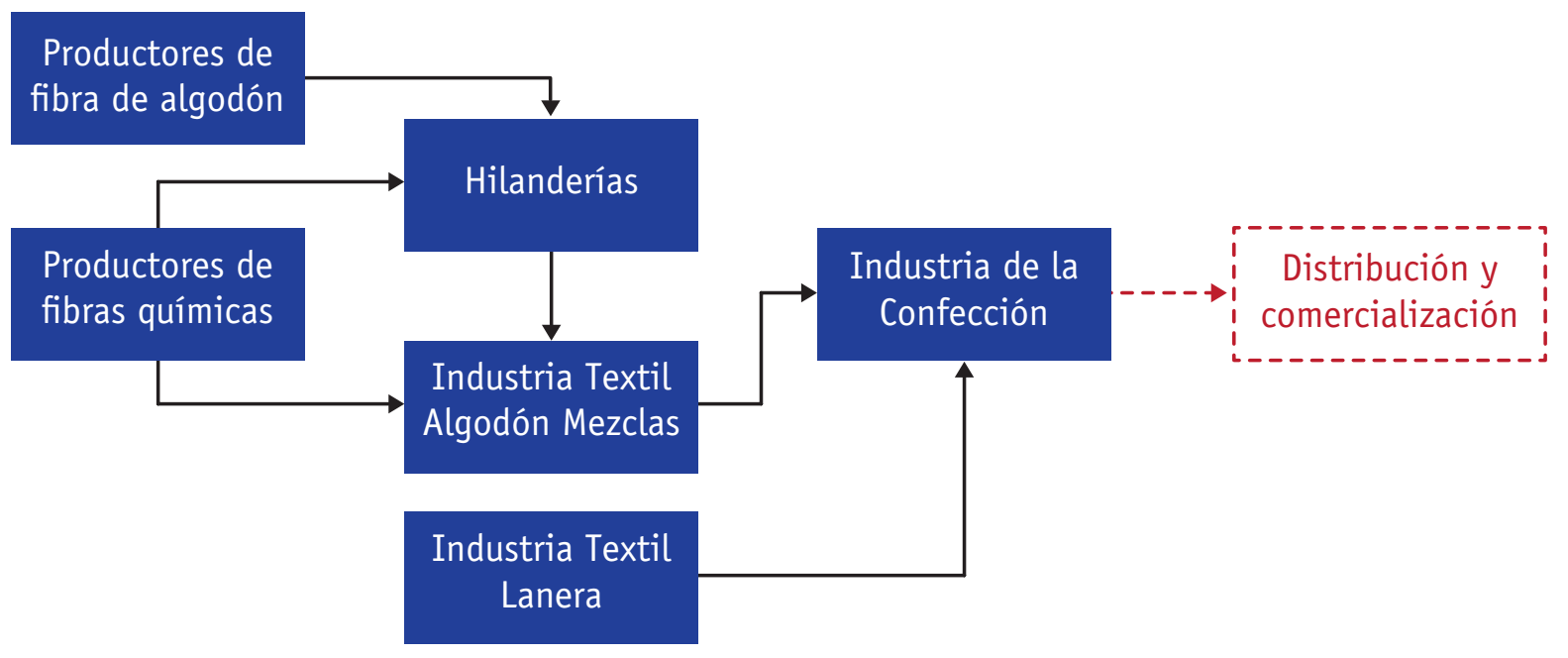

Fuente: (Legis, 2012)

En el caso del departamento de Antioquia (Colombia), el sector textil-confección representa el 30,6\% del empleo industrial del departamento, lo que genera cerca de 109000 empleos en el Valle de Aburra
(Cámara de Comercio De Medellín - CCM, 2017). Las empresas de este sector están interconectadas en un clúster textil para fortalecerse y ser más competitivas, de tal forma que este clúster representa 
el $2,5 \%$ del PIB departamental, y $17 \%$ del valor agregado de la industria antioqueña. La actividad textil-confección es liderada desde el municipio de Medellín y su área metropolitana, en donde se genera el 45,5\% del total de clúster nacional (Semana, 2017).

\section{Logística en el sector confección}

Las empresas del sector textil-confecciones están obligadas a realizar ajustes en su cadena de suministros, para reaccionar con la misma prontitud con la que cambia cada colección. Por tal razón, un sistema Justo a Tiempo (JIT) representa una herramienta de gran valor porque permite a las compañías responder a los clientes con precios cada vez más bajos y con una calidad mayor, y promueve que las unidades de mercancía lleguen justo cuando se requieren. “Entre los conceptos básicos del JIT se resaltan la reducción de desperdicios, de todo aquello que no produce valor agregado, tal y como los productos almacenados, en proceso de inspección, productos con retraso, en espera o defectuosos" (Rojas, Guisao y Cano, 2011, p. 12).

Según cifras del Departamento administrativo Nacional de Estadística (DANE), el sector textil-confecciones representa el 7,5\% del PIB manufacturero y el $3 \%$ del PIB nacional y constituye más del $5 \%$ del total de exportaciones del país (DANE, 2016). Esto ha hecho que se promueva la modernización de las operaciones logísticas y algunas alianzas estratégicas entre las empresas del sector, a fin de lograr ser más competitivas en el mercado. Entre dichas alianzas se encuentran aquellas con socios estratégicos que apoyen algunas actividades de la cadena de suministro, mientras la empresa se dedica a lo que realmente implica sus competencias centrales o core business. Este tipo de alianzas, ya sea con proveedores, distribuidores e incluso fabricantes (maquilas), ha permitido a las empresas colombianas estar en cualquier lugar de país y del mundo en el momento indicado.

\section{RESULTADOS}

Es importante evidenciar las dificultades del sector textil-confección tomando en consideración los diversos factores que intervienen en su productividad y competitividad. Algunos de estos factores están directamente relacionados con el mercado específico de la empresa, sus clientes, proveedores, cadenas de valor y logística. También es importante resaltar que existen amenazas y dificultades del entorno que, según los resultados de las entrevistas realizadas, son: la situación fiscal y financiera del país, las políticas de importación y exportación, la tasa representativa del mercado, los tratados comerciales, el contrabando, el lavado de activos y las constantes reformas tributarias implementadas en los últimos años. Todo esto ha ocasionado que la economía dé indicios de recesión, ya que la contracción de la demanda afecta a todos los sectores industriales y manufactureros.

Con la reciente reforma tributaria implementada por el Ministerio de Hacienda de Colombia en 2016, con la que se pretende aumentar el recaudo y disminuir el déficit fiscal (Dirección de Impuestos y Aduanas Nacionales - DIAN, 2016), inevitablemente los sectores industriales y manufactureros nacionales se han visto afectados en gran medida por el incremento del IVA, el cual pasó de $16 \%$ a $19 \%$ (Secretaria General del Senado, 2016). Esta situación ha ocasionado que los índices de productividad, el consumo y la competitividad disminuyan. De igual manera, la capacidad adquisitiva de la población se redujo, mientras que el incremento salarial en el último año fue del $7 \%$ para los empleados de empresas privadas, y del 6,75 $\%$ para los servidores públicos (Ministerio del Trabajo - MINTRABAJ0, 2017).

Con este aumento en el IVA y en la base gravable de la retención en la fuente, sumado a los fenómenos del contrabando y el lavado de activos, la economía del consumidor colombiano promedio se encuentra notablemente afectada; lo cual trae como consecuencia la disminución en las ventas totales durante el año 2017. En comparación con el mismo periodo del año 2016, se registra una caída en ventas totales en diversos sectores, tales como en la industria de las bebidas $(-10,60 \%)$, minerales no metálicos $(-2,80$ $\%)$, sustancias y productos químicos básicos $(-3,60$ $\%)$, partes, piezas y accesorios para vehículos automotores $(-4,40 \%)$, alimentos $(-0,80 \%)$ y, en especial, la industria de confecciones y acabado de productos textiles e hilatura, con el mayor descenso en ventas totales registrado en junio de 2017 (-15,6\%), con 
una proyección de disminución en ventas a nivel nacional del $21,6 \%$, según datos de la Asociación Nacional de Industriales (ANDI, 2017).

En adición, el sector textil y confecciones se enfrenta a otras amenazas que incluyen el desmonte de aranceles mixtos a la mercancía procedente de países asiáticos como China, India y Pakistán, que ingresa a Colombia desde la zona libre de Colón en Panamá, según la Cámara Colombiana de la Confección y afines (CCCA, 2017), lo que favorece el ingreso de prendas y productos textiles a precios muy bajos y poco alcanzables para el competidor nacional. Esta situación le resta competitividad a la industria colombiana, especialmente a las empresas del sector textil, porque para dar inicio al tejido de una tela se incurren en costos de alrededor de 2,5 dólares por kilo, mientras que en el mercado se adquieren telas terminadas y subfacturadas a 1,5 dólares el kilo, lo que representa un valor inferior al de la fibra que requiere la industria local para producirla (ANDI, 2017, p.16).

\section{DISCUSIÓN}

Con base en la información y cifras anteriores, así como las reportadas durante el año 2017 por la Cámara Colombiana de la Confección; el sector textil-confección es uno de los más afectados, declarándose en aprietos económicos y decrecimiento. Por primera vez en la vigesimoctava versión de la feria Colombiamoda (considerada como una de las principales plataformas de negocios para este sector y clúster textil-confección y moda), no se logró alcanzar la meta presupuestada en expectativas de negocios. Esto generó que el nivel de inversión de los compradores locales se redujera en promedio un $50 \%$, a pesar de que el porcentaje de los compradores potenciales fue mayor que en otras versiones de la feria; y del mismo modo, permite inferir comportamientos prudentes por parte de los comercializadores a la hora de hacer nuevas inversiones (Inexmoda, 2017).

Ante estas dificultades, teniendo presente que el sector de las confecciones presenta demandas estacionales, en las cuales los inventarios deben ser renovados constantemente para satisfacer la deman$\mathrm{da}$, se debe tener convergencia con una planificación estratégica logística que permita que esta mercancía aumente su rotación y genere valor. Cabe resaltar que los niveles de incertidumbre en la demanda, los factores externos a la organización (impuestos, contrabando, lavado de activos, competencia desleal, entre otros) y la baja planificación e integración de la cadena de suministros, refleja que los inventarios no vendidos se conviertan en grandes pérdidas por liquidación de mercancías. Esta situación surge de una necesidad evidente de liquidez a corto plazo; no obstante, la destrucción de valor desde la gestión de las marcas es ineludible, ya que al incurrir en liquidaciones de mercancía basada en disminución de precios y promociones, se afecta considerablemente la diferenciación asociada al branding.

Por lo tanto, ante esta situación es totalmente necesario la aplicación de estrategias logísticas y de gestión de cadenas de abastecimiento, con el objetivo de integrar, optimizar y coordinar los procesos empresariales desde los proveedores de materia prima, hasta el consumidor final, gestionando adecuadamente los pronósticos y realizando un análisis fundamental y de entorno respecto a la situación económica del país, así como la recuperación de la confianza del consumidor y los comercializadores. En este sentido, los miembros de la cadena logística del sector deberán realizar un análisis minucioso de las actividades fundamentales de la logística, en aras de cumplir la promesa de venta al consumidor final y aumentar su productividad.

La implementación de una estructura empresarial guiada por un adecuado enfoque logístico es primordial para cualquier tipo de compañía, tanto para aquellas dedicadas a la prestación de servicios como para las industrias manufactureras que busquen rentabilidad, crecimiento y éxito empresarial. Con este enfoque logístico las empresas pueden desarrollar estrategias que abarquen toda la cadena de suministro, iniciando con un estudio de selección de proveedores de materias primas, hasta llegar a la distribución al cliente para su consumo final, incluyendo la logística inversa. En consecuencia, el sector textil-confección debe superar las problemáticas que se presentan a continuación, las cuales se destacan como resultado de las entrevistas y consultas a expertos. 


\section{Principales problemáticas y retos para el sector textil-confección}

\section{Baja venta y baja rotación}

La carga impositiva ha incrementado los precios de venta de los productos, lo que ocasiona que el consumidor actúe con reservas al comprar, ya que los productos de la canasta familiar se han incrementado, y dejan a un segundo plano la adquisición de prendas de vestir. Esta situación presenta el agravante del exceso de productos por lavado de activos y contrabando, lo que genera una sobreoferta y desencadena precios muy inferiores a los costos de producción de una empresa legal.

\section{Exceso de inventarios}

Una vez se presenta el fenómeno de contracción de la demanda y la baja venta de los productos del sector, el exceso de inventarios y la baja rotación (con productos sujetos a estacionalidades y moda) surgen como una gran problemática logística que urge ser intervenida.

\section{Altos costos en la manufactura}

Debido a la inflación, el incremento del precio del dólar, la carga tributaria y los costos del recurso humano; la rentabilidad se ha visto golpeada y con tendencia a la baja. De igual modo, los costos de infraestructura, maquinaria y equipos impacta directamente el flujo de caja de las empresas por el costo de oportunidad que esto representa.

\section{Impuestos y tributos}

Una vez analizada la información del Banco Mundial, se observa que hay una coincidencia en la información obtenida por los empresarios del sector cuando afirman que la tasa efectiva de tributación sobre las utilidades de las empresas sobrepasa el $70 \%$, lo que significa que un porcentaje excesivo de las ganancias se destina al pago de impuestos. Otra situación que preocupa a los empresarios es el tema relacionado con los acuerdos comerciales con países con arancel cero para el ingreso de mercancía al país.

\section{Altas obligaciones bancarias}

La tasa de usura en Colombia fijada por el Banco de la República es concebida como una de las más altas de Latinoamérica, impactando en gran medida el consumo y el endeudamiento necesario por parte de las empresas. El gravamen a los movimientos financieros (4 por mil) ha presentado una gran problemática porque grava las operaciones financieras de cuentas de ahorros, cuentas corrientes, cuentas de depósito, saldos positivos de tarjetas de crédito, fondos comunes, cheques de gerencia, traslado de fondos, desembolsos de créditos, entre otros; impactando directamente en las utilidades. Esto sumado a la informalidad e ilegalidad marcada por el contrabando y el lavado de activos proliferando las transacciones comerciales en efectivo.

De esta forma, se encuentra como común denominador en las entrevistas y consultas a expertos, que la problemática logística de mayor impacto del sector textil-confección radica en el manejo de inventarios, la rotación, el manejo de las promociones y estímulos para la comercialización. Como segunda problemática, se encuentra el lavado de activos y el contrabando, lo que hace que el mercado base su competitividad en vía precio. Seguido a esto, se encuentran los costos de materias primas (por la tasa representativa del mercado), los costos de producción y el costo asociado a la maquinaria, equipos, infraestructura y reconversión tecnológica. Dada la dinámica de las ventas, los ciclos de facturación y la liquidez, las empresas se ven obligadas al manejo financiero de sus portafolios a través de préstamos bancarios con tasas elevadas, sobregiros y altos índices de endeudamiento.

\section{CONCLUSIÓN}

Es evidente el riesgo del sector textil-confección por las múltiples amenazas del entorno económico, político y social del país. Es por esto que se deberán 
plantear políticas y estrategias a corto, mediano y largo plazo que garanticen al sector unos índices aceptables de productividad y competitividad.

Este estudio permitió identificar que, a pesar de las situaciones adversas del entorno económico, las empresas del sector textil-confección pueden sobrellevar la situación actual e incluso ser más competitivas con una adecuada implementación de actividades fundamentales y de soporte de la logística, las cuales permitirán reducir costos importantes de almacenamiento y transporte.

Desde un enfoque logística, concebido como la integración, sincronización y optimización de los procesos involucrados en la cadena de suministro, existen múltiples estrategias, herramientas y procesos que propenderían por el aumento de la competitividad del sector, entre las cuales se resaltan la planificación de la demanda, gerencia estratégica de inventarios, y esquemas colaborativos.

Dentro de la planificación de la demanda se recomienda que las empresas del sector textil-confección realicen un proceso riguroso con modelos estadísticos diseñados para demandas estacionales relacionadas con la moda. Entre estos modelos de planificación de demanda se resaltan los modelos autorregresivos, ARIMA, suavizamiento lineal, Holt Winter y Brown. La selección del modelo dependerá del comportamiento de la serie de tiempo (ruido, estacionalidades, niveles, ciclos, etc); y en adición, estos análisis estadísticos requerirán de la validación cualitativa de informes sectoriales, análisis de entorno, prospectiva, vigilancia tecnológica e inteligencia competitiva, de manera que la incertidumbre por los cambios externos tenga un menor impacto en la toma de decisiones.

Respecto a la gerencia estratégica de inventarios, se recomienda que esta dependa del modelo de abastecimiento que se deriva de la demanda de la empresa. Dado que los productos del sector textilconfección, diseño y moda tienen una alta rotación por los cambios constantes de la moda, el manejo de los saldos, obsoletos o productos de baja rotación deberán minimizarse para que la liquidez y las finan- zas de la empresa no se vean afectados. Se propone entonces una gerencia estratégica de inventarios que contemple el análisis de confiabilidad en dinero, confiabilidad en referencias y unidades, utilizando escategramas para una categorización de Pareto de sus productos y detallado de la variabilidad y comportamiento del histórico de las ventas; así como implementando matrices de rentabilidad frente a rotación, matrices de Kraljic, entre otras.

Finalmente, resulta ineludible el planteamiento de esquemas colaborativos en la cadena de suministro del sector textil-confección, tanto con proveedores, canales de distribución, clientes y consumidores finales. Herramientas como el CRM (Costumer Relationship Management), el CPFR (Collaborative Planning Forecasting and Replenishment), el SyOP (Sales y Operation Planning) son de gran utilidad en la planificación estratégica y logística, ya que permiten integrar los procesos internos de la organización y sincronizar a socios externos como aliados al core de la organización, buscando la optimización de toda la cadena de suministro.

\section{REFERENCIAS BIBLIOGRÁFICAS}

Asociación Nacional de Industriales - ANDI. (29 de enero de 2017). Competitividad del sector textil confección. Bogotá: ANDI.

Asociación Nacional de Industriales - ANDI. (2017). Encuesta de opinión industrial conjunta. Bogota: ANDI. Recuperado de http://www.andi.com.co/Uploads/ Informe\%20E0IC\%20Junio\%202017.pdf

Bahadır, B. y Büyüközkan, G. (2016). Robot Selection for Warehouse. International Logistics and Supply Chain Congress. Izmir, Turkey: Ege University Faculty.

Ballou, R. (2004). Logística: Administración de la cadena de suministro, Vol. 5. Mexico, DF: Prentice Hall.

Cano, J. A., Panizo, C. A., García, F. H. y Rodríguez, J. E. (2015). Estrategias para el mejoramiento la cadena de suministro del carbón en Norte de Santander, Colombia. Boletín de Ciencias de la Tierra, 1(38), 65-74.

Cámara Colombiana de la Confección y afines - CCCA. (16 de Junio de 2017). Sector textil y confección contribuyó con el 82 \% de la perdida de empleo industrial. Recuperado de Camara Colombiana de la Confección y afines: http://ccca.com.co/sector-textil-y-confecciones-contribuyo-con-el-82-perdida-mpleo 
Gestión logística en el sector textil-confección en colombia: retos y oportunidades de mejora para la competitividad

Cámara de Comercio De Medellín - CCM. (2017). Cluster Textil/Confección, Diseño y Moda "Tejiendo juntos la estrategia". Medellin: Camara de Comercio De Medellín Para Antioquia.

Cooper, W. (2010). Textile and apparel supply chains for the 21st century. Journal of Textile and Apparel, Technology and Management, 6(4).

Departamento Administrativo Nacional de Estadística - DANE. (2016). Producto Interno Bruto -PIB- IV trimestre 2016 y Total 2016. Bogota DC.

Dirección de Impuestos y Aduanas Nacionales - DIAN. (2016). Dirección de Impuestos y Aduanas Nacionales. Recuperado de http://www.dian.gov.co/descargas/ centrales/2017/Abece_Reforma_Tributaria_2016.pdf.

Domingo, L. (2016). The Challenges of Logistics 4.0 for the Supply Chain Management and the Information Technology. Noruega: Norwegian University of science and technology.

Fugate, B. (2010). Logistics Performance: Efficiency, Effectiveness, And Differentiation. United States: Jorunal of Business Logistics.

Hülsmann, T. (2015). Logistics 4.0 and Internet of things. Workshop: Platforms for connected Factories of the Future. Frankfurt.

Inexmoda. (2017). Informe de Cierre Colombiamoda 2017. Medellin. Recuperado de: http://colombiamoda. inexmoda.org.co/informe2017/

Legis. (2012). Textiles y confecciones en Colombia. Recuperado de: https://www.legiscomex.com/
BancoMedios/Documentos\%20PDF/documento-completo-estudio-de-mercado-sector-textil-confeccionescolombia-2012-actualziado-legisocmex.pdf

Mentzer, J. (2004). Fundamentals of Supply Chain Management: Twelve Drivers of Competitive Advantage. The United States: SAGE.

Ministerio del Trabajo - MINTRABAJ0. (2017). Incremento salarial de $6.75 \%$ para servidores públicos acordaron Gobierno y centrales obreras. Bogota, DC: MINTRABAJO.

Ramdass, K. y Pretorius, L. (2011). Implementation of modular manufacturing in the clothing industry in Kwazulu-Natal: A case study. South African Journal of Industrial Engineering, 22(1), 167-181.

Rojas, M., Guisao, E. y Cano, J. A. (2011). Logística Integral: Una propuesta práctica para su negocio. Medellín: Universidad de Medellín.

Secretaria General del Senado. (2016). Puertas abiertas acciones visibles de la secretaria general del senado (Boletín informativo). Bogota. Recuperado de http:// www.secretariasenado.gov.co/index.php/puertasabiertas/1305-puertas-abiertas-acciones-visiblesde-la-secretaria-general-senado-de-la-republica

Vélez De Nicholls, L. (2017, julio). Así se fortalece el clúster textil de Antioquia. Revista Semana. Recuperado de https://www.semana.com/contenidos-editoriales/moda-asi-lo-hacemos/articulo/ situacion-de-las-empresas-de-confeccion-diseno-ymoda-de-antioquia/533688 